\title{
Severe influenza symptoms in celiac disease: implications of CD103- dendritic cells?
}

\author{
Raffaella Mormile ${ }^{1}$
}

Accepted: 7 March 2016/Published online: 12 March 2016

(C) Springer-Verlag Berlin Heidelberg 2016

\section{Dear Editor:}

Patients affected by celiac disease (CD) have been reported to have an increased risk for influenza severe enough to merit hospital admission. $\mathrm{CD}$ is an autoimmune disorder characterized by permanent intolerance to dietary gluten in genetically predisposed individuals. Although CD patients mainly suffer from gastrointestinal symptoms, $\mathrm{CD}$ can involve almost any organ system of the body. It has been stated that osteopontin $(\mathrm{OPN})$ is over-expressed in plasma and intestinal tissue from patients with inflammatory bowel diseases including CD. OPN is a pleiotropic pro-inflammatory cytokine that favors the onset of a large number of autoimmune diseases. Dendritic cells (DCs) are significant for the development of intestinal inflammation. It has been found that a subset of DCs, CD103- DCs, expresses high levels of OPN. The expression of OPN by CD103- DCs has been highlighted to drive intestinal inflammation. CD103- DCs are considered as a source of OPN which can act on them in an autocrine manner. CD103- DCs have been generally depicted as important for the development of inflammation. Conversely, CD103+ DCs are non-inflammatory and express profoundly lower levels of OPN. Interestingly, increases in CD103- DC numbers have been ascertained to be accompanied by concomitant reductions in CD103+ DC numbers. Influenza, commonly called the flu, is a contagious respiratory illness caused by flu viruses. It is a ubiquitous infection with a spectrum ranging from mild to severe. Serious outcomes of influenza

Raffaella Mormile

raffaellamormile@alice.it

1 Division of Pediatrics and Neonatology, Moscati Hospital, Via A. Gramsci, 3, 81031 Aversa, Italy infection can result in being hospitalized and occasionally in death. Such variability in clinical presentation and outcome of patients has not been fully clarified, although a role for the complex interplay among virus features, host immune response, and environmental factors has been proposed. Influenza can cause important lung tissue damage. The establishment of a strong CD8T cell response is considered a key factor in determining the outcome of an influenza infection. It has been found that enhancement of CD8 T cell is able to confer protection against lethal doses of influenza. Moreover, it has been revealed that there is a strong correlation between the number of virus-specific effector CD8 T cells in the lung and viral titer reduction. Similarly, the absence of CD8 T cells has been connected with delayed clearance of the influenza virus, elevated pulmonary viral titers, and increased mortality. Lung CD103+ DCs are considered to be central to the initiation of CD8 T cell responses against influenza viruses promoting viral clearance from the lungs. Intriguingly, OPN has been shown to exacerbate pulmonary damage in influenza-induced lung injury. It has been detected that there is a positive correlation between the OPN serum levels and disease severity in influenza lung injury. Influenza vaccination is a widely accepted practice particularly among the high-risk individuals suffering from certain chronic conditions such as pulmonary and cardiac diseases. All these contentions led us to hypothesize that patients affected by $\mathrm{CD}$ may be at high risk of developing serious flurelated respiratory complications as a result of the interaction between OPN and CD103- DCs leading to a downregulation of $\mathrm{CD} 103+\mathrm{DCs}$ and consequently, to reduced CD8 $\mathrm{T}$ cell responses. Therefore, we suggest that influenza vaccination should be strongly recommended for CD patients because it represents the most effective way to prevent infection and severe outcomes caused by influenza viruses in this condition. 